\title{
Efficient and selective cleavage of the tert-butoxycarbonyl (Boc) group under basic condition
}

\author{
Debendra K. Mohapatra* and Kulbhushan A. Durugkar \\ Division of Organic Chemistry: Technology, National Chemical Laboratory Pune-411008, India \\ E-mail: $\underline{d k m \_77 @ y a h o o . c o m ~}$
}

\begin{abstract}
A simple, efficient and mild method for the selective cleavage of tert-butoxycarbonyl (Boc) from dicarbamates and a carbonyl or aromatic ring in conjugation with the nitrogen atom bearing the Boc-group is described under basic condition.
\end{abstract}

Keywords: $\alpha$-Amino acids, di-t-butylimidodicarbonate, selective deprotection, celogenitins, racemization

\section{Introduction}

The natural and unnatural $\alpha$-amino acids play a central role in chemistry and biology. ${ }^{1}$ In addition, to their key biological roles as components of peptides, proteins, and other natural products, $\alpha$-amino acids are utilized in the pharmaceutical, agrochemical, and related industries. The use of $\alpha$-amino acids and their derivatives solely depend on the protecting group attached to the amine and acid functionality. ${ }^{2}$ Among the various protecting groups for amine functionality, the $t$-butoxycarbonyl (Boc) group is one of the most frequently used in organic synthesis due to its chemical stability to basic and mildly acidic conditions and its ease of removal under specific conditions. ${ }^{3}$ However, selective cleavage of the di-t-butylimidodicarbonate, oftenly found as valuable intermediates in Mitsunobu ${ }^{4}$ and Gabriel type ${ }^{5}$ processes towards the synthesis of several bio-active molecules, protected $\alpha$-amino acids to mono-Boc compounds still lacks efficient and milder methods. Numerous methods are available for the removal of the Bocgroup, ${ }^{7}$ including trifluoroacetic acid, ${ }^{8}$ trimethylsilyl iodide, ${ }^{9}$ hydrochloric acid in ethyl acetate, ${ }^{10}$ ceric ammonium nitrate, ${ }^{11}$ tin tetrachloride, ${ }^{12}$ tetrabutylammonium fluoride, ${ }^{13}$ zinc bromide, ${ }^{14}$ boron trifluoride, ${ }^{15}$ thermolytic removal ${ }^{16}$ etc. However, there are very few methods for the selective cleavage ${ }^{17 \mathrm{a}-\mathrm{f}}$ of the di-Boc protected $\alpha$-amino acids to mono-Boc compounds. Still organic community seeks simple, efficient and milder methods for selective deprotection of the well known protecting groups by preserving the other functionalities. 
During our investigation towards the synthesis of celogenitins, ${ }^{18}$ we tried to perform Suzuki coupling reaction at $\mathrm{C}-2$ position of tryptophan derivative 1a with imidazole in the presence of $\mathrm{Cs}_{2} \mathrm{CO}_{3}$ and catalytic amount of palladium reagent in acetonitrile at $70{ }^{\circ} \mathrm{C}$. It is worthy to mention that, under various conditions of employing different palladium reagent, we could able to isolate only the Boc-deprotected product $\mathbf{1 b}$ (Scheme 1).

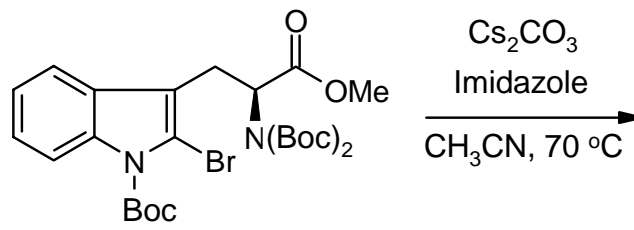

$1 \mathrm{a}$

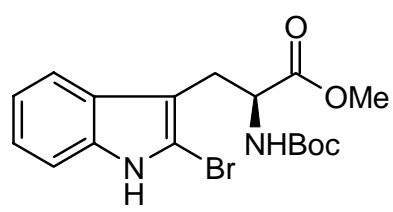

$1 \mathbf{b}$

\section{Scheme 1}

We report herein a new and mild method for the deprotection of Boc-group from amino compounds under basic conditions, using $\mathrm{Cs}_{2} \mathrm{CO}_{3}$ and imidazole system, from di-tbutyldiimidodicarbonate as well as nitrogen atom conjugated to a carbonyl or aromatic system.

\section{Results and Discussion}

To explore the scope and limitations, we investigated a series of Boc-protected amino compounds with different protecting groups and functionalities, which are shown in Table 1. Although it is known that cleavage of the protecting groups in methyl $N$-Boc- $\alpha$-amino esters gave the corresponding $N$-Boc-amino acids without racemization ${ }^{19}$ under basic condition, in our case, we were worried about such a possibility in the presence of an additional Boc group. To investigate this, we submitted compound 2, 3, and 4 to above basic conditions to cleave the Boc group. We found that the optical rotation of the product obtained changed with time, showing clearly that epimerization has occurred. Furthermore, chiral HPLC analysis of $\mathbf{2 b}$ showed two peaks confirming the epimerization during the reaction. ${ }^{20}$ In light of this result, we decided to convert the acid into its benzyl esters. To ensure the enantiomeric purity of such a product (8b), we again submitted for the chiral HPLC which showed single peak at the corresponding retention time and in addition, the rotation clearly confirmed no detectable racemization. With the retention of optical purity on amino acids, we applied the above method to other Boc-protected compounds. Boc-group attached to nitrogen atoms part of an aromatic system or in conjugation with a carbonyl group was removed in high yields. Simple aliphatic mono-Boc compounds were unaffected under above reaction conditions. In entry 12a, where the nitrogen functionality is protected by Boc as well as $\mathrm{Cbz}$ group, we found that only Boc group was selectively cleaved from the nitrogen and $\mathrm{Cbz}$ group was untouched affording the product $\mathbf{1 2 b}$ in $82 \%$ yield. 
Table 1. Selective removal of Boc-group under basic condition

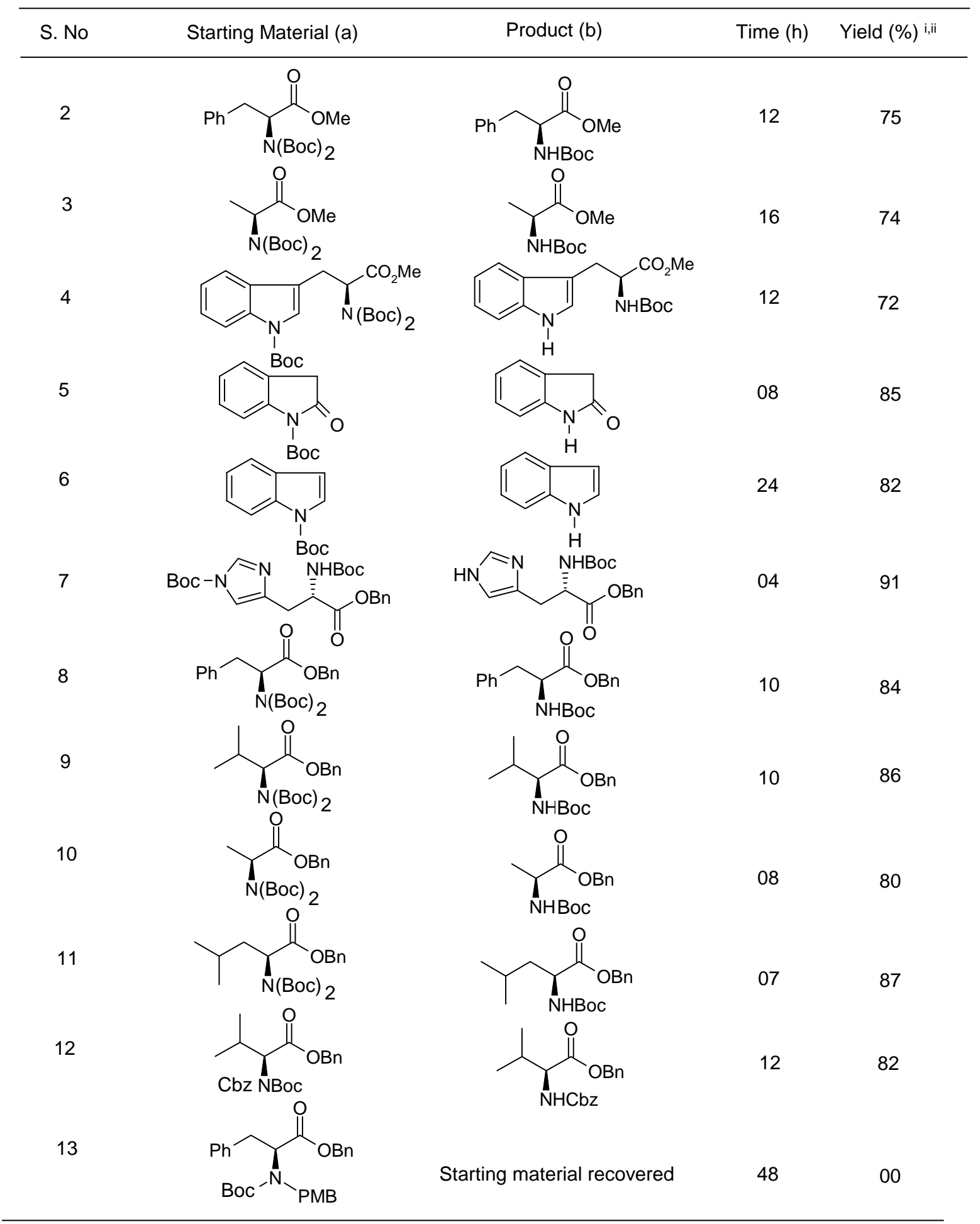

${ }^{i}$ All reactions were carried out in acetonitrile at $70{ }^{\circ} \mathrm{C}$ and the products characterized by NMR, IR, elemental analysis.

${ }^{i i}$ Isolated yield. 
When the nitrogen functionality was doubly protected with an additional protecting group besides Boc (entry 13a), such as p-methoxybenzyl (PMB) group, the deprotection of the Bocgroup was not observed. Interestingly in case of 7a, the $t$-butoxycarbonyl group attached to the ring nitrogen was cleaved selectively compare to the BOC group present on the aliphatic nitrogen atom to afford compound $\mathbf{7 b}$ which is difficult to make directly from histidine in $91 \%$ yield. Next various organic solvents were tried and we found that in particular acetonitrile and THF were the solvents where better results were obtained. Another important point is that both the reagents are required in stoichiometric amount for the completion of the reaction.

The mechanism proposed by Stafford et al. ${ }^{16 \mathrm{~b}}$ and Martin et al. ${ }^{16 \mathrm{f}}$ for the cleavage of $\mathrm{N}$-Boc amines based on the formation of a six-membered chelate seems to be applicable to our method. We proposed that the initial chelation between the metal ion and two oxygen of the carbonyl group followed by abstraction of proton from the methyl group led to the loss of isobutene, $\mathrm{CO}_{2}$ and formation of Boc-deprotected product. Our observation supports the structure A, in which both carbonyls are chelated with the cation.

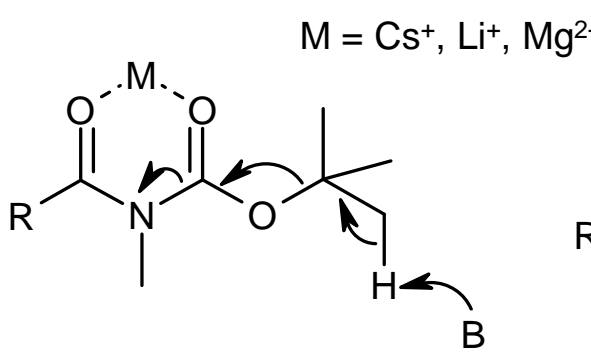

(A)

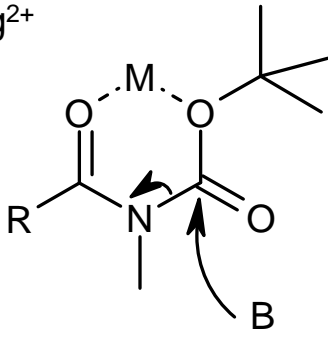

(B)

\section{Conclusions}

In conclusion, we have described a simple, mild, and efficient protocol for the deprotection of the Boc group from Boc-protected carbamates, amides and nitrogen atom present in the aromatic ring under basic condition at $70{ }^{\circ} \mathrm{C}$.

\section{Experimental Section}

General Procedures. Solvents were purified and dried by standard procedures before use. Column chromatography was carried out with silica gel (60-120 mesh). Infrared spectra were recorded with Shimadzu IR 420 and Perkin-Elmer 683 spectrometers. NMR spectra were recorded on Bruker AC-200 and Bruker DRX-500 machine in $\mathrm{CDCl}_{3}$ with TMS as internal standard. Mass spectra were obtained with Finningen MAT 1210 mass spectrometer. Optical rotations were measured with digital polarimeter. Elemental analysis was done on elemental 
analyzer model 1108 EA. All reactions were monitored on $0.25 \mathrm{~mm}$ E-Merck pre-coated silica gel (TLC) plates (60F-254) with UV or $\mathrm{I}_{2}$, anisaldehyde reagent in ethanol. Petroleum ether refers to mixture of hexanes with bp $60-80{ }^{\circ} \mathrm{C}$.

\section{General experimental procedure for Boc deprotection}

To a stirred solution of dicarbamates and a carbonyl or aromatic ring in conjugation with the nitrogen atom bearing the Boc-group (1a-13a) $(1 \mathrm{mmol})$ in acetonitrile $(5 \mathrm{~mL})$, was added $\mathrm{Cs}_{2} \mathrm{CO}_{3}(1.5 \mathrm{mmol})$ and imidazole $(1.5 \mathrm{mmol})$ and the reaction mixture was heated to $70{ }^{\circ} \mathrm{C}$ until the completion of the reaction [monitored by TLC, ethyl acetate: petroleum ether (1:9)]. The reaction mixture was then cooled to room temperature, filtered over celite and concentrated. The viscous liquid was purified by column chromatography (silica gel, ethyl acetate/petroleum ether) affording the product (1b-13b) in 72-91\% yield.

Spectral data of compound 1a. Colorless liquid; $\mathrm{R}_{\mathrm{f}}=0.5$ (5\% ethyl acetate/petroleum ether); ${ }^{1} \mathrm{H}$ NMR $\left(500 \mathrm{MHz}, \mathrm{CDCl}_{3}\right): \delta 8.06(\mathrm{~d}, 1 \mathrm{H}, J=5.7 \mathrm{~Hz}), 7.45(\mathrm{~d}, 1 \mathrm{H}, J=5.7 \mathrm{~Hz}), 7.24(\mathrm{~m}, 1$ $\mathrm{H}), 7.18(\mathrm{~m}, 1 \mathrm{H}), 3.78(\mathrm{~s}, 3 \mathrm{H}), 3.49(\mathrm{~m}, 2 \mathrm{H}), 1.68(\mathrm{~s}, 9 \mathrm{H}), 1.31(\mathrm{~s}, 9 \mathrm{H}), 1.28(\mathrm{~s}, 9 \mathrm{H}) ;{ }^{13} \mathrm{C}$ NMR (125 MHz, $\mathrm{CDCl}_{3}$ ) 170.4, 151.5, 148.8, 148.4, 136.9, 136.5, 129.2, 128.1, 124.4, 122.9, 119.1, 116.7, 82.6, 57.6, 52.2, 28.1, 27.6, 25.9; Anal. Calcd. for $\mathrm{C}_{27} \mathrm{H}_{37} \mathrm{BrN}_{2} \mathrm{O}_{8}$ : C, 54.28; H, 6.24; N, 4.69. Found: C, 54.16; H, 6.08; N, 4.78.

Spectral data of compound $1 \mathrm{~b}$. Colorless liquid; $\mathrm{R}_{\mathrm{f}}=0.5(20 \%$ ethyl acetate/petroleum ether); ${ }^{1} \mathrm{H}$ NMR (200 MHz, $\mathrm{CDCl}_{3}$ ): $\delta 8.43$ (br s, $\left.1 \mathrm{H}\right), 7.43-7.08(\mathrm{~m}, 4 \mathrm{H}), 5.16(\mathrm{~d}, 1 \mathrm{H}, \mathrm{J}=7.6 \mathrm{~Hz})$, $4.63(\mathrm{~m}, 1 \mathrm{H}), 3.70(\mathrm{~s}, 3 \mathrm{H}), 3.19(\mathrm{~m}, 2 \mathrm{H}), 1.41(\mathrm{~s}, 9 \mathrm{H})$; Anal. Calcd. for $\mathrm{C}_{17} \mathrm{H}_{21} \mathrm{BrN}_{2} \mathrm{O}_{4}$ : C, 51.40; H, 5.33; N, 7.05. Found: C, 51.26; H, 5.31; N, 7.16.

Spectral data of compound 2a. Colorless liquid; $\mathrm{R}_{\mathrm{f}}=0.6$ (5\% ethyl acetate/petroleum ether); $[\alpha]_{\mathrm{D}}{ }^{25}=-89.7\left(c 0.96, \mathrm{CHCl}_{3}\right)$; IR (neat): 2979, 2935, 1795, 1744, $1703 \mathrm{~cm}^{-1} ;{ }^{1} \mathrm{H}$ NMR $(200$ $\left.\mathrm{MHz}, \mathrm{CDCl}_{3}\right): \delta 7.18(\mathrm{~m}, 5 \mathrm{H}), 5.14(\mathrm{dd}, 1 \mathrm{H}, J=3.6,7.3 \mathrm{~Hz}), 3.75(\mathrm{~s}, 3 \mathrm{H}), 3.37(\mathrm{dd}, 1 \mathrm{H}, J=$ $3.5,14.2 \mathrm{~Hz}), 3.25(\mathrm{dd}, 1 \mathrm{H}, J=10.2,14.2 \mathrm{~Hz}), 1.38(\mathrm{~s}, 18 \mathrm{H}) ;{ }^{13} \mathrm{C} \mathrm{NMR}\left(50 \mathrm{MHz}, \mathrm{CDCl}_{3}\right)$ $170.5,151.3,137.3,129.2,128.0,126.2,82.5,59.1,51.9,35.9,27.5$; Anal. Calcd. for $\mathrm{C}_{20} \mathrm{H}_{29} \mathrm{NO}_{6}$ : C, 63.31; H, 7.70; N, 3.69. Found: C, 63.48; H, 7.66; N, 3.92.

Spectral data of compound $2 \mathrm{~b}$. Colorless liquid; $\mathrm{R}_{\mathrm{f}}=0.5$ (15\% ethyl acetate/petroleum ether); $[\alpha]_{\mathrm{D}}^{25}=+18.4\left(c\right.$ 1.54, $\left.\mathrm{CHCl}_{3}\right) ; \operatorname{lit}^{17 \mathrm{e}}[\alpha]_{\mathrm{D}}{ }^{25}=+42.5\left(\right.$ c $\left.1.32, \mathrm{CHCl}_{3}\right)$ (prepared by following standard procedure and took the rotation too); IR (neat): 3372, 3029, 2978, 1746, $1715 \mathrm{~cm}^{-1} ;{ }^{1} \mathrm{H}$ NMR (200 MHz, $\left.\mathrm{CDCl}_{3}\right): \delta$ 7.25-7.13 (m, $\left.5 \mathrm{H}\right), 4.94(\mathrm{~d}, 1 \mathrm{H}, J=7.4 \mathrm{~Hz}), 4.59(\mathrm{~m}, 1 \mathrm{H}), 3.71$ (s, $3 \mathrm{H}), 3.07(\mathrm{~m}, 2 \mathrm{H}), 1.41(\mathrm{~s}, 9 \mathrm{H}) ;{ }^{13} \mathrm{C} \mathrm{NMR}\left(50 \mathrm{MHz}, \mathrm{CDCl}_{3}\right)$ 172.0, 154.7, 135.9, 129.0, 128.2, 126.7, 79.3, 54.2, 51.7, 38.0, 28.0; Anal. Calcd. for $\mathrm{C}_{15} \mathrm{H}_{21} \mathrm{NO}_{4}$ : C, 64.50; H, 7.58; N, 5.02. Found: C, 64.78; H, 7.46; N, 4.89 .

Spectral data of compound 3a. Colorless liquid; $\mathrm{R}_{\mathrm{f}}=0.5$ (5\% ethyl acetate/ petroleum ether); $[\alpha]_{\mathrm{D}}{ }^{25}=-39.6\left(\mathrm{c} 1.0, \mathrm{CHCl}_{3}\right)$; IR $\left(\mathrm{CHCl}_{3}\right): 3021,2983,1788,1746,1697 \mathrm{~cm}^{-1} ;{ }^{1} \mathrm{H}$ NMR $(200$ $\mathrm{MHz}, \mathrm{CDCl}_{3}$ ): $\delta$ 4.99-4.89 (q, $\left.1 \mathrm{H}, J=6.82,13.90 \mathrm{~Hz}\right), 3.72(\mathrm{~s}, 3 \mathrm{H}), 1.50(\mathrm{br} \mathrm{s}, 21 \mathrm{H}) ;{ }^{13} \mathrm{C} \mathrm{NMR}$ $\left(50 \mathrm{MHz}, \mathrm{CDCl}_{3}\right)$ 173.0, 151.4, 82.4, 53.5, 51.8, 27.7, 15.3; MS (MALDI TOF): m/z 304.18 (65, $\mathrm{M}^{+}$), 248.12 (100), 192.05 (91), 148.07 (80). Anal. Calcd. for $\mathrm{C}_{14} \mathrm{H}_{25} \mathrm{NO}_{6}$ : C, 55.43; H, 8.31; N, 4.62. Found: C, 55.41; H, 8.29; N, 4.64. 
Spectral data of compound $3 \mathbf{b}$. Colorless liquid; $\mathrm{R}_{\mathrm{f}}=0.2$ (5\% ethyl acetate/petroleum ether); $[\alpha]_{\mathrm{D}}{ }^{25}=-2.75$ (c 2.0, $\left.\mathrm{CHCl}_{3}\right) ; \mathrm{IR}\left(\mathrm{CHCl}_{3}\right): 3440,3378,2981,1744,1709, \mathrm{~cm}^{-1} ;{ }^{1} \mathrm{H}$ NMR $(200$ $\mathrm{MHz}, \mathrm{CDCl}_{3}$ ): $\delta 5.06$ (br s, $\left.1 \mathrm{H}\right), 4.30(\mathrm{~m}, 1 \mathrm{H}), 3.75(\mathrm{~s}, 3 \mathrm{H}), 1.44$ (s, $\left.9 \mathrm{H}\right), 1.38$ (d, $3 \mathrm{H}, J=7.2$ $\mathrm{Hz}$ ); ${ }^{13} \mathrm{C}$ NMR (50 MHz, $\mathrm{CDCl}_{3}$ ) 173.1, 154.5, 78.6, 51.4, 48.5, 27.6, 15.5; MS (MALDI TOF): $\mathrm{m} / \mathrm{z} 226.11\left(26, \mathrm{M}^{+}+\mathrm{Na}\right), 204.12\left(100, \mathrm{M}^{+}\right), 148.07$ (98). Anal. Calcd. for $\mathrm{C}_{9} \mathrm{H}_{17} \mathrm{NO}_{4}: \mathrm{C}, 55.19$; H, 8.43; N, 6.89. Found: C, 55.21; H, 8.41; N, 6.88.

Spectral data of compound $4 a$. $R_{\mathrm{f}}=0.5\left(5 \%\right.$ ethyl acetate/petroleum ether); $[\alpha]_{\mathrm{D}}{ }^{25}=-75.2(\mathrm{c}$ 1.0, $\mathrm{CHCl}_{3}$ ); IR (neat): 3021, 2982, 2932, 1788, 1745, 1739, $1703 \mathrm{~cm}^{-1} ;{ }^{1} \mathrm{H}$ NMR $(200 \mathrm{MHz}$, $\left.\mathrm{CDCl}_{3}\right): \delta 8.10(\mathrm{~d}, 1 \mathrm{H}, J=8.64 \mathrm{~Hz}), 7.51(\mathrm{~d}, 1 \mathrm{H}, J=8.42 \mathrm{~Hz}), 7.35-7.10(\mathrm{~m}, 3 \mathrm{H}), 5.14(\mathrm{dd}, 1$ $\mathrm{H}, J=3.84,11.53 \mathrm{~Hz}), 3.76(\mathrm{~s}, 3 \mathrm{H}), 3.54-3.27(\mathrm{~m}, 2 \mathrm{H}), 1.64(\mathrm{~s}, 9 \mathrm{H}), 1.32(\mathrm{~s}, 18 \mathrm{H}),{ }^{13} \mathrm{C} \mathrm{NMR}$ $\left(50 \mathrm{MHz}, \mathrm{CDCl}_{3}\right) 170.1,151.3,149.0,135.1,130.1,123.9,123.8,122.1,118.5,116.0,114.8$, 82.7, 82.2, 57.7, 51.8, 27.7, 27.3, 25.1; Anal. Calcd. for $\mathrm{C}_{27} \mathrm{H}_{38} \mathrm{~N}_{2} \mathrm{O}_{8}$ : C, 62.53; H, 7.39; N, 5.40. Found: C, 62.36; H, 7.48; N, 5.62 .

Spectral data of compound $4 \mathrm{~b}$. Colorless liquid; $\mathrm{R}_{\mathrm{f}}=0.4(10 \%$ ethyl acetate/petroleum ether); $[\alpha]_{\mathrm{D}}{ }^{25}=+39.3\left(c \mathrm{1} 1.0, \mathrm{CHCl}_{3}\right)$; IR (neat): 3478, 3433, 3019, 2981, 2954, 1739, $1705 \mathrm{~cm}^{-1} ;{ }^{1} \mathrm{H}$ NMR $\left(500 \mathrm{MHz}, \mathrm{CDCl}_{3}\right): \delta 8.45($ br s, $1 \mathrm{H}), 7.55(\mathrm{~d}, 1 \mathrm{H}, J=8.50 \mathrm{~Hz}), 7.25(\mathrm{~d}, 1 \mathrm{H}, J=8.44$ Hz), $7.15(\mathrm{~m}, 1 \mathrm{H}), 7.10(\mathrm{~m}, 1 \mathrm{H}), 6.90(\mathrm{~s}, 1 \mathrm{H}), 5.01(\mathrm{~m}, 1 \mathrm{H}), 4.60(\mathrm{~m}, 1 \mathrm{H}), 3.65(\mathrm{~s}, 3 \mathrm{H}), 3.25$ (m, $2 \mathrm{H}), 1.41$ (s, $9 \mathrm{H}) ;{ }^{13} \mathrm{C}$ NMR $\left(125 \mathrm{MHz}, \mathrm{CDCl}_{3}\right)$ 172.7, 155.2, 136.3, 122.8, 122.1, 119.5, 118.6, 111.2, 110.0, 80.0, 54.4, 52.0, 28.4; Anal. Calcd. for $\mathrm{C}_{17} \mathrm{H}_{22} \mathrm{~N}_{2} \mathrm{O}_{4}: \mathrm{C}, 64.14 ; \mathrm{H}, 6.96 ; \mathrm{N}$, 8.79. Found: C, 64.29; H, 7.38; N, 8.81.

Spectral data of compound 5a. Light yellow oil; $\mathrm{R}_{\mathrm{f}}=0.5$ ( $5 \%$ ethyl acetate/ petroleum ether); IR $\left(\mathrm{CHCl}_{3}\right): 3021,2930,1789,1766,1730, \mathrm{~cm}^{-1} ;{ }^{1} \mathrm{H}$ NMR $\left(200 \mathrm{MHz}, \mathrm{CDCl}_{3}\right): \delta 7.80(\mathrm{~d}, 1 \mathrm{H}, J$ $=8.84 \mathrm{~Hz}), 7.32-7.11(\mathrm{~m}, 3 \mathrm{H}), 3.64(\mathrm{~s}, 2 \mathrm{H}), 1.04(\mathrm{~s}, 9 \mathrm{H}) ;{ }^{13} \mathrm{C} \mathrm{NMR}\left(50 \mathrm{MHz}, \mathrm{CDCl}_{3}\right) 172.2$, 148.9, 140.9, 127.7, 123.8, 122.9, 114.8, 83.6, 36.1, 27.8; Anal. Calcd. for $\mathrm{C}_{13} \mathrm{H}_{15} \mathrm{NO}_{3}$ : C, 66.94; H, 6.48; N, 6.00. Found: C, 66.91; H, 6.39; N, 5.98.

Spectral data of compound 6a. Colorless liquid; $\mathrm{R}_{\mathrm{f}}=0.4$ (4\% ethyl acetate/ petroleum ether); IR $\left(\mathrm{CHCl}_{3}\right): 2979,2933,1729, \mathrm{~cm}^{-1} ;{ }^{1} \mathrm{H} \mathrm{NMR}\left(200 \mathrm{MHz}, \mathrm{CDCl}_{3}\right): \delta 8.12(\mathrm{~d}, 1 \mathrm{H}, J=7.96 \mathrm{~Hz})$, 7.58-7.50 (m, $2 \mathrm{H}), 7.33-7.16(\mathrm{~m}, 2 \mathrm{H}), 6.54(\mathrm{~d}, 1 \mathrm{H}, J=3.66 \mathrm{~Hz}), 1.67(\mathrm{~s}, 9 \mathrm{H}) ;{ }^{13} \mathrm{C}$ NMR $(50$ $\left.\mathrm{MHz}, \mathrm{CDCl}_{3}\right)$ 149.4, 135.2, 130.5, 125.5, 123.9, 122.4, 120.7, 115.0, 107.0, 83.0, 27.9; MS (MALDI TOF): m/z $240.11\left(100, \mathrm{M}^{+}+\mathrm{Na}\right), 218.09\left(10, \mathrm{M}^{+}\right), 197.12$ (96), 162.07 (49), 141.05 (41), 126.10 (98), 108.09 (34). Anal. Calcd. for $\mathrm{C}_{13} \mathrm{H}_{15} \mathrm{NO}_{2}$ : C, 71.87; H, 6.96; N, 6.45. Found: C, 71.83; H, 6.95; N, 6.41.

Spectral data of compound 8a. Colorless liquid; $\mathrm{R}_{\mathrm{f}}=0.6$ ( $5 \%$ ethyl acetate /petroleum ether); $[\alpha]_{\mathrm{D}}{ }^{25}=-56.6$ (c 2.45, $\left.\mathrm{CHCl}_{3}\right)$; IR (neat): $3021,2981,1787,1742,1708 \mathrm{~cm}^{-1} ;{ }^{1} \mathrm{H}$ NMR $(200$ $\left.\mathrm{MHz}, \mathrm{CDCl}_{3}\right): \delta 7.22(\mathrm{~m}, 10 \mathrm{H}), 5.19(\mathrm{~m}, 3 \mathrm{H}), 3.40(\mathrm{dd}, 1 \mathrm{H}, J=5.05,14.02 \mathrm{~Hz}), 3.26(\mathrm{dd}, 1 \mathrm{H}$, $J=10.23,14.02 \mathrm{~Hz}), 1.35(\mathrm{~s}, 18 \mathrm{H}) ;{ }^{13} \mathrm{C}$ NMR $\left(50 \mathrm{MHz}, \mathrm{CDCl}_{3}\right) 170.0,151.6,137.5,135.5$, 129.4, 128.3, 128.2, 128.0, 126.4, 82.6, 66.7, 59.3, 35.8, 27.7; MS (MALDI TOF): $\mathrm{m} / \mathrm{z} 478.25$ $\left(83, \mathrm{M}^{+}+\mathrm{Na}\right), 456.27\left(100, \mathrm{M}^{+}\right), 400.21$ (86),344.14 (49), 274.20 (48), 252.15 (54). Anal. Calcd for $\mathrm{C}_{26} \mathrm{H}_{33} \mathrm{NO}_{6}$ : C, 68.55; H, 7.30; N, 3.08. Found: C, 68.74; H, 7.49; N, 3.42.

Spectral data of compound $8 \mathrm{~b}$. White crystalline solid, $\mathrm{mp}=64.9{ }^{\circ} \mathrm{C} ; \mathrm{R}_{\mathrm{f}}=0.6(10 \%$ ethyl acetate /petroleum ether); $[\alpha]_{\mathrm{D}}{ }^{25}=+14.9\left(\right.$ c 1.00, $\left.\mathrm{CHCl}_{3}\right)$; IR (neat): 3438, 3019, 2981, 1737, 
$1711 \mathrm{~cm}^{-1} ;{ }^{1} \mathrm{H}$ NMR $\left(500 \mathrm{MHz}, \mathrm{CDCl}_{3}\right): \delta$ 7.34-7.04 (m, $\left.10 \mathrm{H}\right), 5.14(\mathrm{dd}, 2 \mathrm{H}, J=13.88,24.67$ $\mathrm{Hz}), 4.96(\mathrm{~m}, 1 \mathrm{H}), 4.62(\mathrm{~m}, 1 \mathrm{H}), 3.08(\mathrm{~m}, 2 \mathrm{H}), 1.41(\mathrm{~s}, 9 \mathrm{H}) ;{ }^{13} \mathrm{C} \mathrm{NMR}\left(125 \mathrm{MHz}, \mathrm{CDCl}_{3}\right)$ 171.4, 154.7, 135.9, 135.2, 129.1, 128.2, 128.1, 126.7, 79.4, 66.7, 54.4, 38.1, 28.1; MS (MALDI TOF): m/z 378.18 (100, $\left.\mathrm{M}^{+}+\mathrm{Na}\right), 356.20\left(44, \mathrm{M}^{+}\right), 274.20$ (9), 252.15(11), 208.14 (16). Anal. Calcd. for $\mathrm{C}_{21} \mathrm{H}_{25} \mathrm{NO}_{4}$ : C, 70.96; H, 7.09; N, 3.94. Found: C, 70.58; H, 7.35; N, 3.68.

Spectral data of compound 9a. Colorless liquid; $\mathrm{R}_{\mathrm{f}}=0.4$ (5\% ethyl acetate/ petroleum ether); $[\alpha]_{\mathrm{D}}{ }^{25}=-32.9\left(\mathrm{c} 1.0, \mathrm{CHCl}_{3}\right)$; IR $\left(\mathrm{CHCl}_{3}\right): 3021,2982,1786,1744,1698 \mathrm{~cm}^{-1} ;{ }^{1} \mathrm{H}$ NMR $(200$ $\left.\mathrm{MHz}, \mathrm{CDCl}_{3}\right): \delta 7.31(\mathrm{~s}, 5 \mathrm{H}), 5.14(\mathrm{dd}, 2 \mathrm{H}, J=12.63,23.12 \mathrm{~Hz}) 4.52(\mathrm{~d}, 1 \mathrm{H}, J=9.35 \mathrm{~Hz})$, 2.58-2.40 (m, $1 \mathrm{H}), 1.44(\mathrm{~s}, 18 \mathrm{H}), 1.16(\mathrm{~d}, 3 \mathrm{H}, J=6.44 \mathrm{~Hz}), 0.88(\mathrm{~d}, 3 \mathrm{H}, J=6.95 \mathrm{~Hz}) ;{ }^{13} \mathrm{C}$ NMR (50 MHz, $\left.\mathrm{CDCl}_{3}\right)$ 169.7, 152.1, 135.6, 128.0, 127.6, 127.5, 82.2, 65.9, 63.1, 27.5, 21.8, 18.6; MS (MALDI TOF): m/z 430.24 (6, $\mathrm{M}^{+}+\mathrm{Na}$ ), 408.25 (10, $\left.\mathrm{M}^{+}\right), 352.19$ (9),274.19 (8), 208.14 (8), 126.10 (98), 126.10 (100). Anal. Calcd. for $\mathrm{C}_{22} \mathrm{H}_{33} \mathrm{NO}_{6}$ : C, 64.84; H, 8.16; N, 3.44. Found: C, 64.79; H, 8.18; N, 3.42.

Spectral data of compound $9 \mathrm{~b}$. Colorless liquid; $\mathrm{R}_{\mathrm{f}}=0.2$ (5\% ethyl acetate/petroleum ether); $[\alpha]_{\mathrm{D}}{ }^{25}=-3.02\left(\mathrm{c} 2.1, \mathrm{CHCl}_{3}\right)$; IR $\left(\mathrm{CHCl}_{3}\right): 3442,2972,1744,1712,1501 \mathrm{~cm}^{-1} ;{ }^{1} \mathrm{H}$ NMR $(200$ $\left.\mathrm{MHz}, \mathrm{CDCl}_{3}\right): \delta 7.34(\mathrm{~s}, 5 \mathrm{H}), 5.16(\mathrm{dd}, 2 \mathrm{H}, J=12.25,19.58 \mathrm{~Hz}), 5.00(\mathrm{~d}, 1 \mathrm{H}, J=8.08 \mathrm{~Hz})$, 4.29-4.22 (m, $1 \mathrm{H}), 2.22-2.10(\mathrm{~m}, 1 \mathrm{H}), 1.44$ (s, $9 \mathrm{H}), 0.93$ (d, $3 \mathrm{H}, J=6.82 \mathrm{~Hz}), 0.84$ (d, $3 \mathrm{H}, J$ $=6.94 \mathrm{~Hz}) ;{ }^{13} \mathrm{C} \mathrm{NMR}\left(50 \mathrm{MHz}, \mathrm{CDCl}_{3}\right)$ 171.8, 155.3, 135.3, 128.3, 128.1, 79.2, 66.5, 58.3, 30.0, 28.1, 18.8, 17.3; MS (MALDI TOF): m/z $330.18\left(10, \mathrm{M}^{+}+\mathrm{Na}\right), 308.19\left(15, \mathrm{M}^{+}\right), 304.19$ (24), 252.13 (18), 248.12 (73), 204.13 (69), 192.06 (100). Anal. Calcd. for $\mathrm{C}_{17} \mathrm{H}_{25} \mathrm{NO}_{4}$ : C, 66.43; $\mathrm{H}$, 8.20 ; N, 4.56. Found: C, 66.40; H, 8.18; N, 4.53.

Spectral data of compound 10a. White powder, $\mathrm{mp}=51.9{ }^{\circ} \mathrm{C} ; \mathrm{R}_{\mathrm{f}}=0.5(5 \%$ ethyl acetate /petroleum ether); $[\alpha]_{\mathrm{D}}{ }^{25}=-21.8\left(c 1.25, \mathrm{CHCl}_{3}\right)$; IR (neat): 2980, 2941, 1794, 1750, $1701 \mathrm{~cm}^{-1}$; ${ }^{1} \mathrm{H}$ NMR $\left(500 \mathrm{MHz}, \mathrm{CDCl}_{3}\right): \delta 7.34(\mathrm{~m}, 5 \mathrm{H}), 5.16(\mathrm{~s}, 2 \mathrm{H}), 5.02(\mathrm{q}, 1 \mathrm{H}, J=7.33,13.92 \mathrm{~Hz})$, $1.54(\mathrm{~d}, 3 \mathrm{H}, J=7.33 \mathrm{~Hz}), 1.46(\mathrm{~s}, 18 \mathrm{H}) ;{ }^{13} \mathrm{C} \mathrm{NMR}\left(125 \mathrm{MHz}, \mathrm{CDCl}_{3}\right)$ 170.8, 151.8, 135.6, 128.3, 127.8, 82.8, 66.6, 53.8, 27.8, 15.2; MS (MALDI-TOF): m/z 402.21 (7, $\left.\mathrm{M}^{+}+\mathrm{Na}\right), 380.22$ $\left(100, \mathrm{M}^{+}\right), 324.16(80), 280.17$ (34), 224.10 (43), 180.11 (74). Anal. Calcd. for $\mathrm{C}_{20} \mathrm{H}_{29} \mathrm{NO}_{6}$ : C, 63.31; H, 7.70; N, 3.69. Found: C, 63.16; H, 8.16; N, 3.60.

Spectral data of compound 10b. Colorless thick liquid; $\mathrm{R}_{\mathrm{f}}=0.5(10 \%$ ethyl acetate /petroleum ether); $[\alpha]_{\mathrm{D}}{ }^{25}=-11.2$ (c 1.35, EtOH); IR (neat): 3367, 3066, 3034, 2979, 2935, 1742, $1716 \mathrm{~cm}^{-1}$; ${ }^{1} \mathrm{H}$ NMR (200 MHz, $\left.\mathrm{CDCl}_{3}\right): \delta 7.35(\mathrm{~m}, 5 \mathrm{H}), 5.18(\mathrm{~d}, 2 \mathrm{H}, J=4.61 \mathrm{~Hz}), 4.35(\mathrm{~m}, 1 \mathrm{H}), 1.44$ (s, $9 \mathrm{H}), 1.42(\mathrm{~d}, 3 \mathrm{H}, J=7.76 \mathrm{~Hz}) ;{ }^{13} \mathrm{C} \mathrm{NMR}\left(50 \mathrm{MHz}, \mathrm{CDCl}_{3}\right)$ 172.9, 154.9, 135.4, 128.3, 127.9, 79.5, 66.6, 49.2, 28.1, 18.1; MS (MALDI TOF): m/z $302.21\left(100, \mathrm{M}^{+}+\mathrm{Na}\right), 280.17\left(51, \mathrm{M}^{+}\right)$, 252.14 (10), 224.10 (14), 180.11 (12). Anal. Calcd. for $\mathrm{C}_{15} \mathrm{H}_{21} \mathrm{NO}_{4}$ : C, 64.49; H, 7.58; N, 5.02. Found: C, 64.25; H, 7.54; N, 4.90 .

Spectral data of compound 11a. Colorless syrup; $\mathrm{R}_{\mathrm{f}}=0.4(10 \%$ ethyl acetate/ petroleum ether); $[\alpha]_{\mathrm{D}}{ }^{25}=-30.1\left(\mathrm{c} 1.0, \mathrm{CHCl}_{3}\right)$; IR $\left(\mathrm{CHCl}_{3}\right): 3019,2962,1728,1711,1500 \mathrm{~cm}^{-1} ;{ }^{1} \mathrm{H}$ NMR $(200$ $\left.\mathrm{MHz}, \mathrm{CDCl}_{3}\right): \delta 7.31(\mathrm{~s}, 5 \mathrm{H}), 5.14(\mathrm{dd}, 2 \mathrm{H}, J=12.50,15.79 \mathrm{~Hz}) 4.94(\mathrm{dd}, 1 \mathrm{H}, J=5.69,8.85)$, 1.93-1.84 (m, $1 \mathrm{H}), 1.69-1.61(\mathrm{~m}, 1 \mathrm{H}), 1.44(\mathrm{~s}, 18 \mathrm{H}), 0.93(\mathrm{~d}, 6 \mathrm{H}, J=6.57 \mathrm{~Hz}) ;{ }^{13} \mathrm{C}$ NMR $(50$ $\left.\mathrm{MHz}, \mathrm{CDCl}_{3}\right)$ 171.1, 152.2, 128.4, 127.9, 82.7, 66.6, 56.6, 38.4, 27.9, 25.1, 23.3, 21.8; MS 
(MALDI TOF): m/z 444.26 (7, $\left.\mathrm{M}^{+}+\mathrm{Na}\right), 422.26\left(4, \mathrm{M}^{+}\right), 326.17$ (9), 304.18 (41), 248.12 (100).Anal. Calcd. for $\mathrm{C}_{23} \mathrm{H}_{35} \mathrm{NO}_{6}$ : C, 65.54; H, 8.37; N, 3.32. Found: C, 65.51; H, 8.35; N, 3.39. Spectral data of compound 11b. Colorless liquid; $\mathrm{R}_{\mathrm{f}}=0.2(10 \%$ ethyl acetate/ petroleum ether); $[\alpha]_{\mathrm{D}}{ }^{25}=-13.5\left(\mathrm{c} 2.0, \mathrm{CHCl}_{3}\right)$; IR $\left(\mathrm{CHCl}_{3}\right): 3441,3021,1784,1740,1698 \mathrm{~cm}^{-1} ;{ }^{1} \mathrm{H} \mathrm{NMR}$ $\left(200 \mathrm{MHz}, \mathrm{CDCl}_{3}\right): \delta 7.34(\mathrm{~s}, 5 \mathrm{H}), 5.15(\mathrm{dd}, 2 \mathrm{H}, J=12.38,15.53 \mathrm{~Hz}) 4.90(\mathrm{~d}, 1 \mathrm{H}, J=8.34)$, 4.40-4.29 (m, $1 \mathrm{H}), 1.69-1.47$ (m, $3 \mathrm{H}), 1.43$ (s, $9 \mathrm{H}), 0.93$ (d, $3 \mathrm{H}, J=1.89 \mathrm{~Hz}), 0.90$ (d, $3 \mathrm{H}, J=$ $2.14 \mathrm{~Hz}) ;{ }^{13} \mathrm{C} \mathrm{NMR}\left(50 \mathrm{MHz}, \mathrm{CDCl}_{3)}\right.$ 172.9, 155.1, 135.4, 128.3,128.0, 127.9, 79.3, 66.5, 52.1, 41.4, 28.1, 24.5, 22.6, 21.7; MS (MALDI TOF): m/z 344.20 (6, $\left.\mathrm{M}^{+}+\mathrm{Na}\right), 322.21\left(4, \mathrm{M}^{+}\right), 304.18$ (53), 248.18 (97), 204.13 (38), 192.05 (99), 148.07 (100).Anal. Calcd. for $\mathrm{C}_{18} \mathrm{H}_{27} \mathrm{NO}_{4}$ : C, 67.26; H, 8.47; N, 4.36. Found: C, 67.24; H, 8.45; N, 4.38.

Spectral data of compound 12a. Colorless liquid; $\mathrm{R}_{\mathrm{f}}=0.3(10 \%$ ethyl acetate/ petroleum ether); $[\alpha]_{\mathrm{D}}{ }^{25}=-29.1\left(\mathrm{c} 1.0, \mathrm{CHCl}_{3}\right)$; IR $\left(\mathrm{CHCl}_{3}\right): 3031,2971,1791,1747,1701 \mathrm{~cm}^{-1} ;{ }^{1} \mathrm{H}$ NMR $\left(200 \mathrm{MHz}, \mathrm{CDCl}_{3}\right): \delta 7.29(\mathrm{~m}, 10 \mathrm{H}), 5.15(\mathrm{~d}, 2 \mathrm{H}, J=1.01 \mathrm{~Hz}), 5.07(\mathrm{~d}, 2 \mathrm{H}, J=8.59 \mathrm{~Hz}) 4.60$ $(\mathrm{d}, 1 \mathrm{H}, J=9.35), 2.58-2.40(\mathrm{~m}, 1 \mathrm{H}), 1.38(\mathrm{~s}, 9 \mathrm{H}), 1.16(\mathrm{~d}, 3 \mathrm{H}, J=6.57 \mathrm{~Hz}), 0.84(\mathrm{~d}, 3 \mathrm{H}, J=$ $6.94 \mathrm{~Hz}) ;{ }^{13} \mathrm{C}$ NMR $\left(50 \mathrm{MHz}, \mathrm{CDCl}_{3}\right)$ 169.4, 153.72, 151.3, 135.5, 135.1, 128.2, 128.0, 127.8, 127.7, 83.0, 68.3, 66.2, 63.2, 28.2, 27.6, 27.5, 21.9, 18.6; MS (MALDI TOF): m/z 464.22 (100, $\left.\mathrm{M}^{+}+\mathrm{Na}\right), 442.25\left(46, \mathrm{M}^{+}\right), 386.19$ (53), 342.19(40). Anal. Calcd. for $\mathrm{C}_{25} \mathrm{H}_{31} \mathrm{NO}_{6}$ : C, 68.01; $\mathrm{H}$, 7.08; N, 3.17. Found: C, 67.98; H, 7.10; N, 3.15.

Spectral data of compound 12b. Colorless thick liquid; $\mathrm{R}_{\mathrm{f}}=0.4(20 \%$ ethyl acetate/ petroleum ether); $[\alpha]_{\mathrm{D}}{ }^{25}=-2.7\left(\mathrm{c} 1.1, \mathrm{CHCl}_{3}\right)$; IR $\left(\mathrm{CHCl}_{3}\right): 3434,3358,3032,2966,1724,1512 \mathrm{~cm}^{-1} ;{ }^{1} \mathrm{H}$ NMR (200 MHz, $\left.\mathrm{CDCl}_{3}\right): \delta 7.33(\mathrm{~m}, 10 \mathrm{H}), 5.29(\mathrm{~d}, 1 \mathrm{H}, J=8.84 \mathrm{~Hz}), 5.15(\mathrm{~d}, 2 \mathrm{H}, J=4.17$ Hz), 5.09 (s, $2 \mathrm{H}), 4.37-4.31(\mathrm{dd}, J=4.67,9.09 \mathrm{~Hz}), 2.25-2.09(\mathrm{~m}, 1 \mathrm{H}), 0.93$ (d, $3 \mathrm{H}, J=6.82$ $\mathrm{Hz}), 0.84(\mathrm{~d}, 3 \mathrm{H}, J=6.85 \mathrm{~Hz}) ;{ }^{13} \mathrm{C} \mathrm{NMR}\left(50 \mathrm{MHz}, \mathrm{CDCl}_{3}\right)$ 171.4, 155.8, 136.0, 135.0, 127.8, 127.8, 127.5, 127.4, 66.2, 58.6, 30.6, 18.4, 17.0; MS (MALDI TOF): m/z 364.16 (100, $\left.\mathrm{M}^{+}+\mathrm{Na}\right)$, 342.18 (11, M ${ }^{+}$), 304.19 (28), 248.12(42), 192.06 (45). Anal. Calcd. for $\mathrm{C}_{20} \mathrm{H}_{23} \mathrm{NO}_{4}$ : C, 70.36; $\mathrm{H}$, $6.79 ; \mathrm{N}, 4.10$. Found: C, 70.39; H, 6.75; N, 4.08.

\section{Acknowledgements}

KAD thanks CSIR, New Delhi for award of Senior Research Fellowship. We are grateful to Dr. M. K Gurjar, Head, Organic Chemistry: Technology Division for his constant encouragement and support.

\section{References}

1. Wagner, I.; Musso, H. Angew. Chem., Int. Ed. 1983, 22, 816.

2. Prakash, I.; Schaad, D. R. Chem. Rev. 1996, 96, 835.

3. (a) Gunnarsson, K.; Grehn, L.; Ragnarsson, U. Angew. Chem., Int. Ed. 1988, 27, 400. (b) Carpino, L. A.; Mansour, E. A. E.; El-Faham, A. J. Org. Chem. 1993, 58, 4162. 
4. Koppel, I.; Koppel, J.; Degerback, F.; Ragnarsson, U. J. Org. Chem. 1991, 56, 7172. (b) Campbell, J. A.; Hart, D. J. J. Org. Chem. 1993, 58, 2900.

5. Gibson, M, S.; Bradshaw, R. W. Angew. Chem., Int. Ed. 1968, 7, 919. (b) Ragnarsson, U.; Grehn, L. Acc. Chem. Res. 1991, 24, 285.

6. Sugawara, N.; Stevens, E. S.; Bonora, G. M.; Toniolo, C. J. Am. Chem. Soc. 1980, 102, 7044. (b) Burk, M. J.; Allen, J. G. J. Org. Chem. 1997, 62, 7054. (c) Kakotos, G.; Padron, J. M.; Martin, T.; Gibbsons, W. A.; Martin, V. S. J. Org. Chem. 1998, 63, 3741.

7. Green, T. W.; Wuts, P. G. M. In Protective Groups in Organic Synthesis; Wiley: New York, 1999; pp-518-525 and references therein.

8. Sakai, N.; Ohfune, I. J. J. Am. Chem. Soc. 1992, 114, 998.

9. Lott, R. S.; Chauhan, V. S.; Stammer, C. H. J. Chem. Soc., Chem. Commun. 1979, 495-.

10. Stahl, G. L.; Walter, R.; Smith, C. W. J. Org. Chem. 1978, 43, 2285.

11. Hwu, J. R.; Jain, M. L.; Tsay, S. C.; Hakimelahi, G. H. Tetrahedron Lett. 1996, 37, 2035.

12. Frank, R.; Schutkowski, M. Chem Commun. 1996, 2509.

13. Routier, S.; Sauge, L.; Ayrebe, N.; Coudert, G.; Merour, J. Y. Tetrahedron Lett. 2002, 43, 589.

14. Nigam, S. C.; Mann, A.; Taddei, M.; Wermuth, C. G. Synth. Commun. 1989, 19, 3139.

15. Evans, E. F.; Lewis, N. J.; Kapfer, I.; Macdonald, G.; Taylor, R. J. K. Synth. Commun. 1997, $27,1819$.

16. Rawal, V. H.; Cava, M. P. Tetrahedron Lett. 1985, 26, 6141.

17. (a) Van Benthem, R. A. T. M.; Hiemstra, H.; Speckamp, W. N. J. Org. Chem. 1992, 57, 6083. (b) Stafford, J. A.; Brackeen, M. F.; Karanewsky, D. S.; Valvano, N, L. Tetrahedron Lett. 1993, 34, 7873. (c) Burkhart, F.; Hoffmann, M.; Kessler, H. Angew. Chem., Int. Ed. 1997, 36, 1191. (d) Yadav, J. S.; Reddy, B. V. S.; Reddy, K. S. Synlett, 2002, 468. (e) Yadav, J. S.; Reddy, B. V. S.; Reddy, K. S. Tetrahdron Lett. 2002, 43, 1549. (f) Hernandez, J. N.; Ramirez, M. A.; Martin, V. S. J. Org. Chem. 2003, 68, 743. (g) Nakamura, K.; Nakajima, T.; Kayahara, H.; Nomura, E.; Taniguchi, H. Tetrahedron Lett. 2004, 45, 495. (h) Tom, N. J.; Simon, W. M.; Frost, H. N.; Ewing, M. Tetrahedron Lett. 2004, 45, 905.

18. Suzuki, H.; Morita, H.; Iwasaki, S.; Kobayashi, J. Tetrahedron 2003, 59, 5307 and references therein.

19. Bodansky, M.; Bodansky, A. The Practice of Peptide Synthesis; Springer-Verlag: Berlin, 1984; p 177.

20. Enatiomeric purity of the product $\mathbf{2 b}$ formed was verified by HPLC analysis. HPLC conditions: column, Chiralcel OD-25 cm; mobile phase, petroleum ether:IPA (90:10); flow rate, $1 \mathrm{ml} / \mathrm{min}$; UV detection at $254 \mathrm{~nm}$ (Retention time for racemic $\mathbf{2 b}$ prepared by mixture of D- and L-phenyl alanine is 6.983 and 7.594 where as the product obtained from reaction showed retention time at 7.054 and 7.650; HPLC conditions: column, Chiralcel OD-25 cm; mobile phase, petroleum ether:IPA (98:2); flow rate, $1 \mathrm{ml} / \mathrm{min}$; UV detection at $254 \mathrm{~nm}$ retention time for racemic $\mathbf{8 b}$ prepared by following standard procedure is 9.008 (D-phenyl alanine) and 9.908 (L-phenyl alanine) and the product from the reaction mixture showed at 10.106 . 